\title{
171. Vortex Rings in Transformer Oil Produced by Electric Discharge.
}

\author{
By Yoshiro IkedA and Etsuro Kato.
}

Faculty of Engineering, Hokkaido Imperial University.

(Rec. 27 Oct. 1928. Comm. by T. Terada, M.I.A., 2 Nov. 1928.)

From the researches ${ }^{1)}$ on the peculiar phenomena of electric discharge on dielectric plate, we are inclined to consider that both the circular spark and the radial discharge figure at low pressure of gas may be caused by some vortex motion of the medium surrounding the dielectrics.

From this reason, we have attempted to produce some vortices of transformer oil on a plate glass. The apparatus is the same, in principle, as the one used in the case of the circular spark, and shown in Fig. 1.

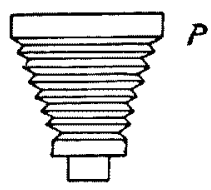

Fig. 1.

$\mathrm{T}:$ High tension transformer.

$\mathrm{S}$ : Spark gap.

$\mathrm{N}:$ Needle.

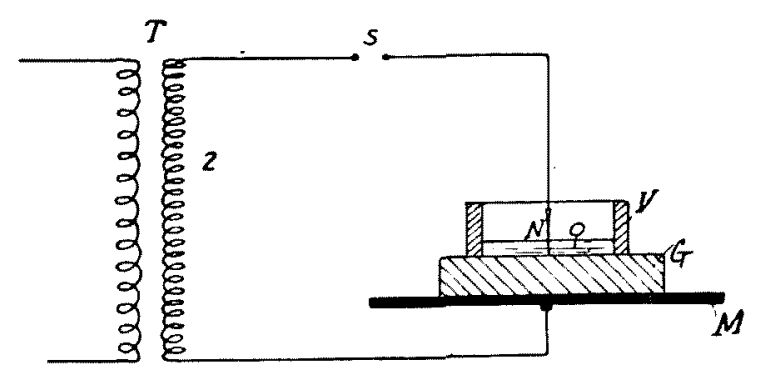

$\mathrm{O}$ : Transformer oil.

$\mathrm{G}$ : Plate glass.

$\mathrm{M}$ : Metal plate.

$\mathrm{P}$ : Photographic camera.

1) Y. Ikeda, T. Itoh and S. Kogima, Circular spark on the dielectric plate, Proc., 3 (1927), 13. Memoir of the Faculty of Engineering, Hokkaido, Japan, 1 (1927), 105.

T. Itoh, Further Researches on the Circular Spark, Memoir of the Faculty of Engineering, Hokkaido, Japan, 1 (1927), 113. Air., Proc., 4 (1928), 16.

T. Itoh, Electric Discharge Figure on Dielectric Plate at Low Pressure of

T. Itoh, Radial Electric Discharge Figure on Dielectric Plate at Low Pressure of Gas, Memoir of the Faculty of Engineering, Hokkaido, Japan, 1 (1928), 237.

T. Itoh, Parallel Striated Figure of Electric Discharge on Dielectric Plate at Low Pressure of Air, Proc., 4 (1928), 286. 
By varying the spark gap, the terminal voltage of transformer and the depth of transformer oil, we obtained some vortical motion, of which the direction of the axis of vorticity is generally perpendicular to the plate glass, as shown in Fig. 2. $a$.

By a further fine adjustment, we have succeeded in producing circular rings of oil. The motion of the oil in the ring was clearly seen by air bubbles in the oil, as shown in Fig. 2. $b$.

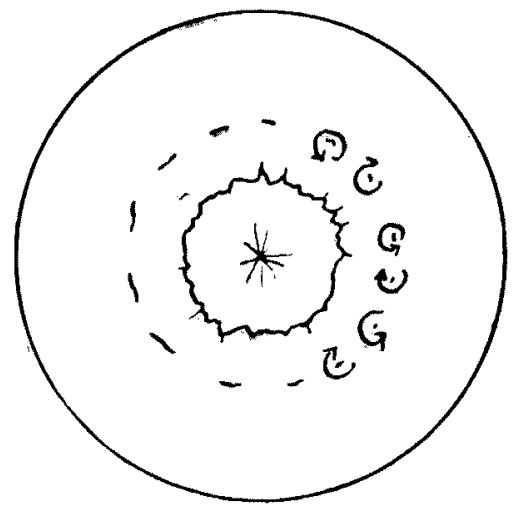

$a$

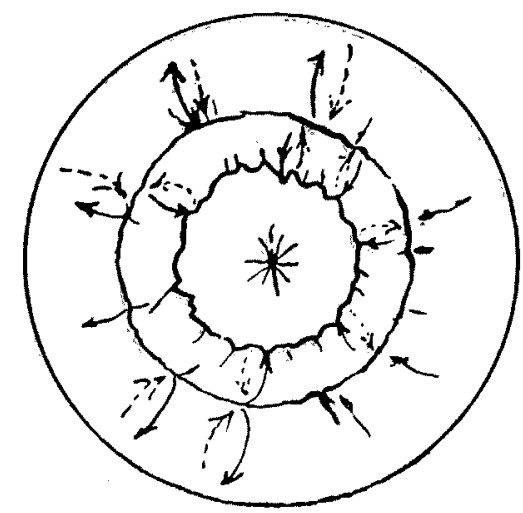

$b$

Fig. 2

The two photographs reproduced in Fig. 4 were taken by the reflected light of magnesium flash and show perspectively the smallest and the intermediate circular rings. Except these two, we have also taken photographs from the upper side with exposures of some seconds, whence we have measured the diameter of the circle at which the two concentric rings are in contact with each other.

It is remarkable that we can see, sometimes, two or three concentric circles at the same time, and that the diameter of the circle is independent of the size of the vessel, while it seems to depend on the thickness of the plate glass. The latter relation is shown in Fig. 3 ; it might be represented by four curves, but quantitative conclusion should be left to further researches. 


\begin{tabular}{|c|c|c|c|c|c|c|c|}
\hline $\begin{array}{c}\text { Thickness } \\
\text { of Plate } \\
\text { Glass. }\end{array}$ & $\begin{array}{c}\text { Depth } \\
\text { of } \\
\text { Oil. }\end{array}$ & $\begin{array}{c}\text { Diameter } \\
\text { of } \\
\text { Vessel. }\end{array}$ & $\begin{array}{c}\text { Diameter } \\
\text { of } \\
\text { Circle. }\end{array}$ & $\begin{array}{c}\text { Thickness } \\
\text { of Plate } \\
\text { Glass. }\end{array}$ & $\begin{array}{c}\text { Depth } \\
\text { of } \\
\text { Oil. }\end{array}$ & $\begin{array}{c}\text { Diameter } \\
\text { of } \\
\text { Vessel. }\end{array}$ & $\begin{array}{c}\text { Diameter } \\
\text { of } \\
\text { Circle. }\end{array}$ \\
\hline $\begin{array}{l}m m . \\
3\end{array}$ & $\begin{array}{l}m m . \\
2.5\end{array}$ & $\underset{94}{m m}$ & $\begin{array}{l}m m \\
62\end{array}$ & $\begin{array}{l}m m \\
38\end{array}$ & $\begin{array}{l}m m . \\
5.0\end{array}$ & $\begin{array}{l}m m . \\
120\end{array}$ & $57^{m m}$ \\
\hline 3 & 2.5 & 120 & 47 & 38 & 5.0 & 120 & 64 \\
\hline 3 & 2.5 & 120 & 49 & 38 & 5.0 & 120 & 65 \\
\hline 3 & 2.5 & 120 & 56 & 38 & 5.0 & 120 & $52,67,100$ \\
\hline 4.5 & 2.5 & 94 & 25 & 38 . & 5.0 & 120 & 63 \\
\hline 6 & 2.0 & 94 & 76 & 49 & 4.0 & 96 & 44 \\
\hline 9 & 2.0 & 94 & 29 & 49 & 4.0 & 96 & 65 \\
\hline 9 & 2.0 & 94 & 29 & 49 & 3.0 & 150 & 65 \\
\hline 9 & 3.5 & 120 & 53,78 & 49 & 3.0 & 150 & 65 \\
\hline 9 & 3.5 & 120 & 57,76 & 49 & 3.0 & 210 & 65 \\
\hline 9 & 3.5 & 120 & 86 & 49 & 5.0 & 210 & 65 \\
\hline 10 & 4.0 & 94 & 55.5 & 69 & 3.5 & 96 & 45 \\
\hline 10 & 4.0 & 94 & 45 & 69 & 4.0 & 120 & 45,62 \\
\hline 10 & 4.0 & 120 & 45 & 69 & 4.0 & 120 & 78 \\
\hline 10 & 4.0 & 120 & 57.5 & 69 & 4.0 & 150 & 78 \\
\hline 10 & 3.0 & 130 & 57.5 & 69 & 4.0 & 150 & 78 \\
\hline 10 & 3.0 & 130 & $57.5,99$ & 73.5 & 3.0 & 94 & 47.5 \\
\hline 10 & 3.0 & 150 & 41 & 73.5 & 3.0 & 94 & 62 \\
\hline 10 & 3.0 & 150 & 82 & 73.5 & 5.0 & 94 & 47.5 \\
\hline 10 & 5.0 & 150 & 45 & 73.5 & 3.0 & 130 & 62 \\
\hline 10 & 5.0 & 150 & 91 & 73.5 & 3.0 & 130 & 39 \\
\hline 10 & 6.0 & 150 & 45 & 73.5 & 4.5 & 130 & 62 \\
\hline 24 & 6.0 & 125 & 52 & 73.5 & 5.0 & 150 & 47.5 \\
\hline 24 & 6.0 & 125 & 52 & 73.5 & 2.5 & 150 & 62 \\
\hline 24 & 6.0 & 125 & 27.5 & 73.5 & 3.0 & 150 & 62 \\
\hline 38 & 5.0 & 120 & 70 & & & & \\
\hline
\end{tabular}




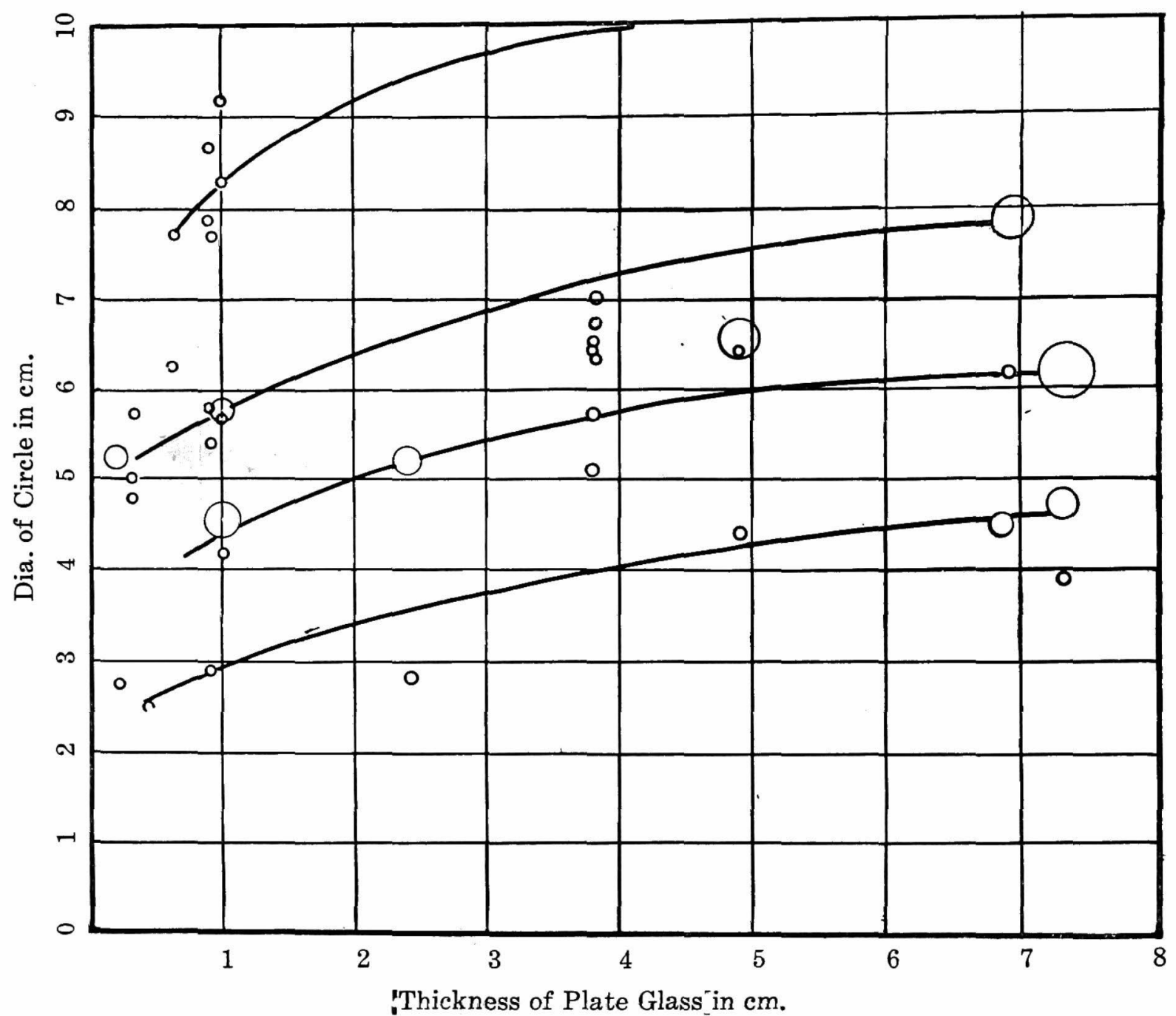

Fig. 3
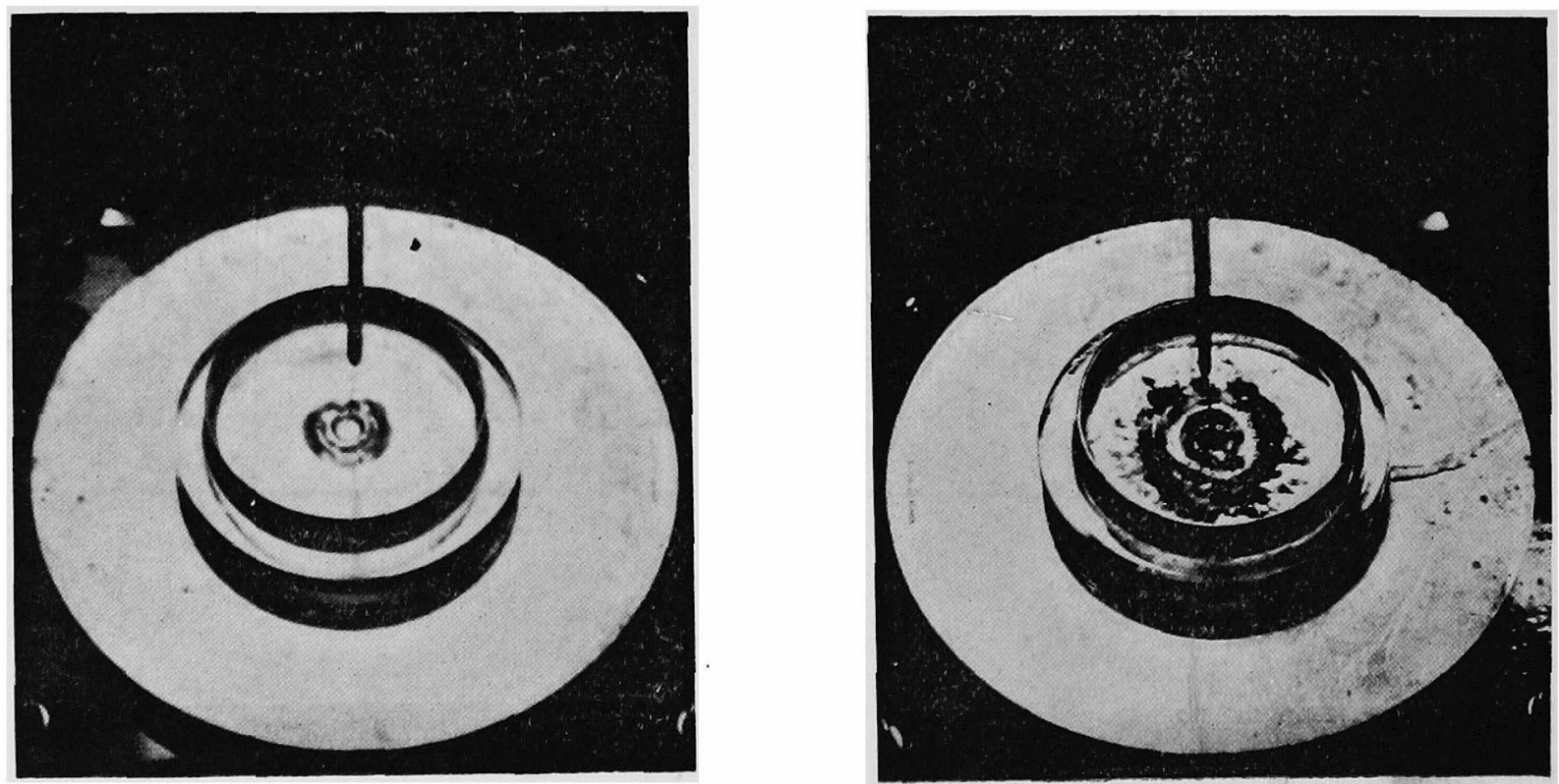

Fig. 4 